\title{
Age-related differences in chest radiographic staging of sarcoidosis in Japan
}

\begin{abstract}
To the Editor:
Sarcoidosis is generally thought to occur more frequently in adults $<40$ years of age, with incidence peaking in patients aged between 20 and 29 years [1]. In Japan, as well as in Europe, there is a second peak in females $>50$ years of age $[2,3]$. When comparing the results of national epidemiological surveys conducted in Japan between 1960 and 2004, the incidence rate in females >50 years of age can be seen to have gradually increased while that for bilateral hilar lymphadenopathy (BHL) has decreased [3]. However, whether there is any association between these trends in Japan remains to be elucidated. An earlier study conducted in Europe suggested that older patients are less likely to present with stage 1 sarcoidosis at diagnosis [2]. Against this background, in this study, we aimed to identify the age-related differences in chest radiographic staging of sarcoidosis in Japan.
\end{abstract}

We reviewed 588 consecutive Japanese patients newly diagnosed with sarcoidosis (431 biopsy proven and 157 clinically proven) who were admitted to or underwent bronchoscopy between April 1974 and July 2012 at Jichi Medical University Hospital (Tochigi, Japan). The diagnosis was made based on the diagnostic criteria and guidelines for sarcoidosis developed by the Japanese Society of Sarcoidosis and Other Granulomatous Disorders in 2007 [4]. We compared chest radiographic staging between subgroups differentiated by sex and age at diagnosis (younger diagnosis group $<45$ years of age, older diagnosis group $\geqslant 45$ years of age) using the Chi-squared test. Significance was set at $\mathrm{p}<0.05$. This study was reviewed and approved by the Jichi Medical University institutional review board (number Rin-A12-54; January 8, 2013).

The radiographs taken closest to the time of diagnosis were staged according to the modified SCADDING [5] criteria: stage 0 , no visible intrathoracic findings; stage 1 , BHL without pulmonary infiltration; stage 2, BHL accompanied by pulmonary infiltration; stage 3, pulmonary infiltration without BHL; and stage 4, advanced pulmonary fibrosis. The radiographs were read by each attending physician and general consensus on radiographic staging was reached in clinical meetings.

The study population consisted of 204 males and 384 females, with 275 assigned to the younger diagnosis group and 313 to the older diagnosis group. BHL findings and lung field abnormalities (stage 2 plus stage $3 / 4$ ) were detected in $61.8 \%$ and $37.1 \%$ of all patients, respectively. Figure 1 shows a comparison of the proportion of patients at each radiographic stage between the groups. Comparison by sex (fig. 1a) showed that in males, stage 1 plus stage 2 disease (Chi squared 4.10, $\mathrm{p}=0.0428$ ) was more common. Comparison by age (fig. 1b) revealed that in the younger group, stage 1 (Chi-squared 7.05, $\mathrm{p}=0.0079$ ), stage 2 (Chi squared 20.9, $\mathrm{p}<0.0001$ ) and stage 1 plus stage 2 (Chi-squared 42.5, $\mathrm{p}<0.0001$ ) disease and BHL findings (Chisquared 32.7, $\mathrm{p}<0.0001$ ) were more common, whereas in the older group, stages 0 and $3 / 4$ disease (Chisquared $17.7(\mathrm{p}<0.0001)$ and Chi-squared $10.8(\mathrm{p}=0.0010)$, respectively) were more common. In terms of age distribution (fig. 1c-e), the proportion of patients with stage 1 or 2 disease decreased consistently with increasing age.

This is the first epidemiological study to demonstrate age-related differences in chest radiographic staging at the time of diagnosis of sarcoidosis in Japan. In the younger diagnosis group, stage 1 and 2 disease was more common, whereas in the older diagnosis group, stage 0 and 3/4 disease was more common. The proportion of patients with stage 1 or 2 disease, which reflects patients presenting with BHL, was clearly associated with age.

Sarcoidosis is characterised by an amplified and persistent T-helper cell (Th) type 1 granulomatous immune response against an unidentified antigen. From the results of molecular biological, pathological and epidemiological studies, it has been presumed that the antigen enters via the lungs during the early stage of the disease $[6,7]$ and affects the regional intrathoracic lymph nodes. Likely carriers of the triggering antigen are mycobacteria and propionibacteria that act as intracellular parasites causing respiratory infection $[8,9]$. Dendritic cells, with the apparent ability to change from and to macrophages, are presumed to digest the antigen, migrate to the lymph node and induce antigen-specific Th1 cell proliferation [8, 10]. Disequilibrium between the local Th1 response and immune regulatory mechanism is considered to account in part for the pathophysiology of sarcoidosis. 


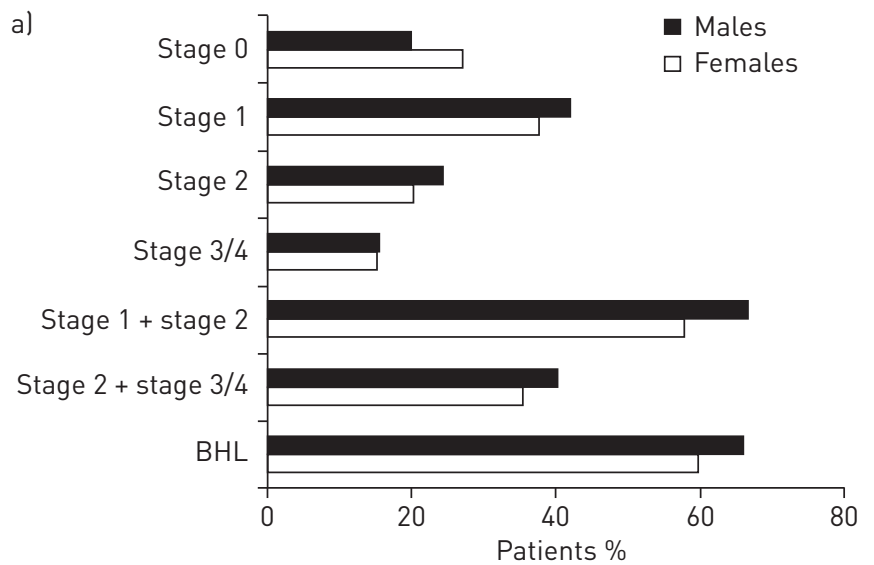

Stage $0 \square$ Stage $1 \square$ Stage $2 \square$ Stage 3/4

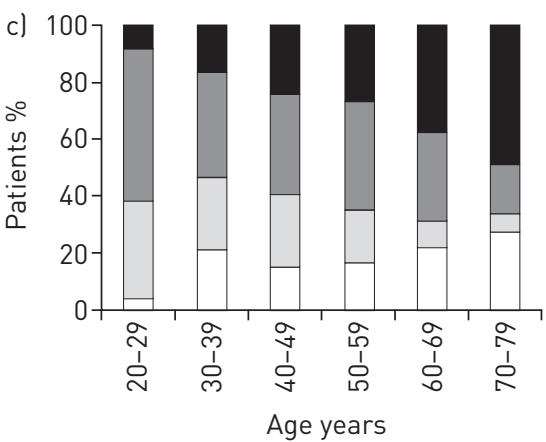

b)

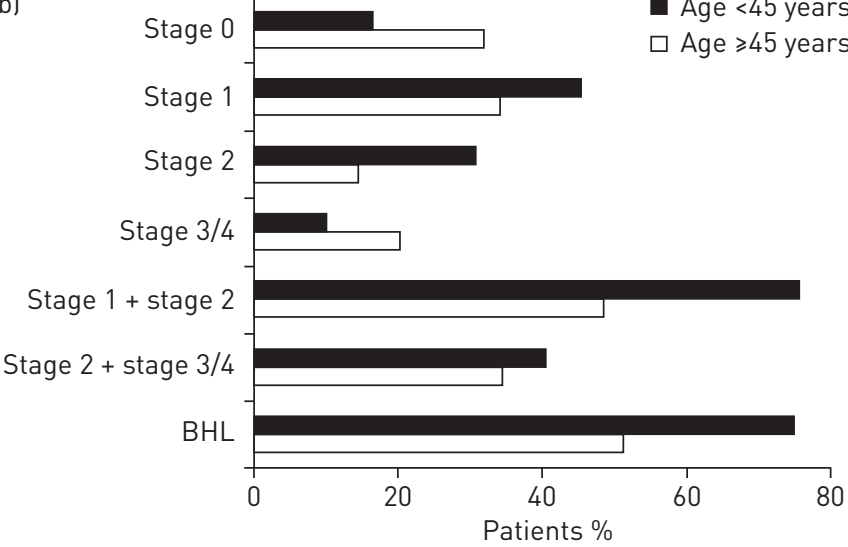

Stage $0 \square$ Stage $1 \square$ Stage $2 \square$ Stage $3 / 4$

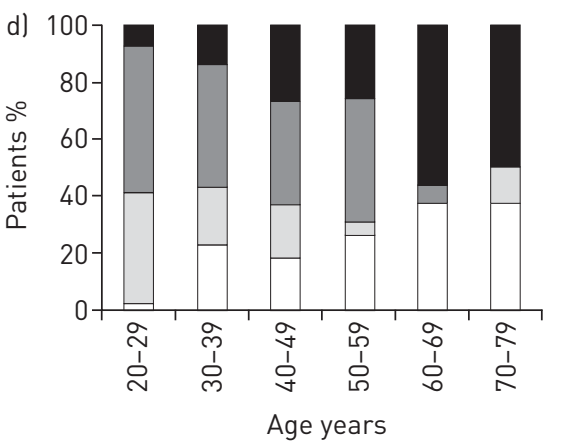

Stage $0 \square$ Stage $1 \square$ Stage $2 \square$ Stage 3/4

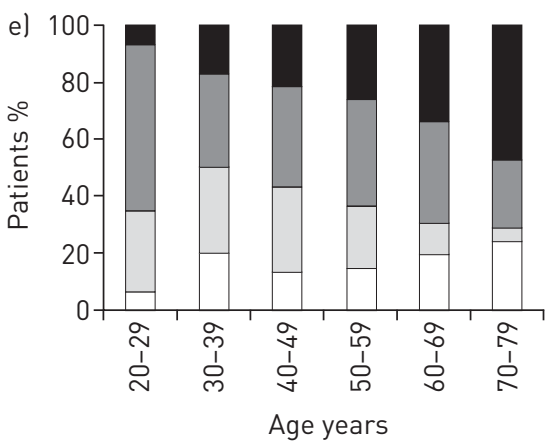

FIGURE 1 Comparison of proportion of patients with radiographic stage between groups on the basis of a) sex and b) age at diagnosis. In males, stage 1 plus stage 2 was more common. In the younger diagnosis group, stage 1 , stage 2 , stage 1 plus stage 2 and bilateral hilar lymphadenopathy (BHL) were more common, while in the older diagnosis group, stages 0 and $3 / 4$ disease were more common. In terms of age distribution, the proportion of patients with stage 1 or 2 disease decreased consistently with increasing age in c) all patients, d) males and e) females.

When considering the present observations, the consistently decreasing occurrence of BHL with increasing age might be explained by the age-associated changes in the proportion of T-cell compartments, a major determinant of the progressive decline in the immune responses with aging. In old age, decreased proportions of nave and effector T-cells [11] and an increased proportion of regulatory T-cells (Tregs), which are likely to accumulate in the lymph nodes, are usually observed [12]. The molecular mechanisms of the Treg-mediated suppression of the immune responses have not been completely elucidated. However, in the presence of Tregs, the arrest of antigen-specific effector T-cells interacting with dendritic cells is reported to be markedly diminished in the lymph nodes [13], which suppress $\mathrm{T}$ cell proliferation programmes. Tregs, which accumulate in the affected lymph nodes as well as in the granuloma periphery of sarcoidosis patients, are reported to have reduced function and to efficiently suppress nave T-cell proliferation but not fully suppress tumour necrosis factor- $\alpha$ secretion; they also do not control local inflammation [14]. This proposed mechanism is compatible with the following description included in a statement on sarcoidosis reported in 1999 [1]: an acute onset with BHL usually heralds a self-limiting course, whereas an insidious onset, especially without pulmonary lesions, may be followed by relentless, progressive fibrosis of the lungs.

The main strength of this study is its consecutive series of patients over four decades. Limitations include some potential selection biases. In particular, subjects were a limited number of patients who visited the respiratory centre of a single university hospital in Tochigi prefecture, and thus the results might not be generalisable to the Japanese population.

In conclusion, the proportion of patients presenting with BHL was associated with patient age, and the recent decreasing trend in the occurrence of BHL in Japan might reflect increasing age at the time of diagnosis of sarcoidosis. 

increasing age http://ow.ly/tybI4

Michiru Sawahata ${ }^{1}$, Yukihiko Sugiyama ${ }^{1}$, Yosikazu Nakamura ${ }^{2}$, Masayuki Nakayama ${ }^{1}$, Naoko Mato ${ }^{1}$, Hideaki Yamasawa ${ }^{1}$ and Masashi Bando ${ }^{1}$

${ }^{1}$ Division of Pulmonary Medicine, Dept of Medicine, Jichi Medical University, Shimotsuke, Tochigi, and ${ }^{2}$ Dept of Public Health, Jichi Medical University, Shimotsuke, Tochigi, Japan.

Correspondence: M. Sawahata, Division of Pulmonary Medicine, Jichi Medical University, 3311-1 Yakushiji, Shimotsukeshi, Tochigi 329-0498, Japan. E-mail: d1114@jichi.ac.jp

Received: Jan 082014 | Accepted after revision: Jan 312014 | First published online: March 132014

Support statement: This study was partly supported by a grant to the Diffuse Lung Diseases Research Group from the Ministry of Health, Labour and Welfare, Japan (grant number H23-Nanchi-Ippan-023). M. Sawahata thanks the Japanese Society of Sarcoidosis and Other Granulomatous Disorders for awarding the Grant for Young Investigators (2013), and Jichi Medical University for awarding the JMU Graduate Student Start-Up Grant for Young Investigators (2012).

Conflict of interest: Disclosures can be found alongside the online version of this article at www.erj.ersjournals.com

\title{
References
}

Statement on sarcoidosis. Am J Respir Crit Care Med 1999; 160: 736-755.

Hillerdal G, Nöu E, Osterman K, et al. Sarcoidosis: epidemiology and prognosis. A 15-year European study. Am Rev Respir Dis 1984; 130: 29-32.

Morimoto T, Azuma A, Abe S, et al. Epidemiology of sarcoidosis in Japan. Eur Respir J 2008; 31: 372-379. [Japanese Society of Sarcoidosis and Other Granulomatous Disorders (JSSOG) diagnostic criteria and guidelines for sarcoidosis - 2006.]. Jpn J Sarcoidosis Other Granulomatous Disorders 2007; 22: 89-101.

5 Scadding JG. Prognosis of intrathoracic sarcoidosis in England. A review of 136 cases after five years' observation. Br Med J 1961; 2: 1165-1172.

6 Müller-Quernheim J. Sarcoidosis: immunopathogenetic concepts and their clinical application. Eur Respir J 1998; 12: 716-738.

7 Corrin B, Nicholson AG, Dewar A. Pathology. In: Mitchell D, Wells A, Spiro S, et al, eds. Sarcoidosis. London, Hodder Arnold, 2012; pp. 41-47.

$8 \quad$ Valeyre D, Prasse A, Nunes H, et al. Sarcoidosis. Lancet 2014; 383: 1155-1167.

9 Negi M, Takemura T, Guzman J, et al. Localization of Propionibacterium acnes in granulomas supports a possible etiologic link between sarcoidosis and the bacterium. Mod Pathol 2012; 25: 1284-1297.

10 Zaba LC, Smith GP, Sanchez M, et al. Dendritic cells in the pathogenesis of sarcoidosis. Am J Respir Cell Mol Biol 2010; 42: 32-39.

11 Linton PJ, Haynes L, Tsui L, et al. From nave to effector-alterations with aging. Immunol Rev 1997; 160: 9-18.

12 Sharma S, Dominguez AL, Lustgarten J. High accumulation of T regulatory cells prevents the activation of immune responses in aged animals. J Immunol 2006; 177: 8348-8355.

13 Shevach EM. Mechanisms of Foxp $3^{+}$T regulatory cell-mediated suppression. Immunity 2009; 30: 636-645.

14 Miyara M, Amoura Z, Parizot C, et al. The immune paradox of sarcoidosis and regulatory T cells. J Exp Med 2006; 203: $359-370$.

\section{Whole-body magnetic resonance imaging in extrathoracic sarcoidosis}

\author{
To the Editor:
}

The course of sarcoidosis is heterogeneous, and the assessment of pulmonary and extrathoracic organ involvement is important for clinical treatment decisions [1]. Whole-body imaging techniques have been evaluated to assess total disease activity $[2,3] .{ }^{18} \mathrm{~F}$-fluoro-2-deoxy-D-glucose positron emission tomography $\left({ }^{18} \mathrm{FDG}-\mathrm{PET}\right)$ and ${ }^{18} \mathrm{FDG}-\mathrm{PET} / \mathrm{computed}$ tomography (CT) allow a complete picture of active intra- and extrapulmonary sites [4]. Whole-body magnetic resonance imaging (WB-MRI) is an established diagnostic tool for multifocal disorders such as multiple myeloma and metastatic diseases [5, 6]. The role of WB-MRI in the assessment of extrathoracic organ involvement in patients with sarcoidosis has not yet been studied.

We present an institutional review board-approved study including 24 patients with histologically confirmed sarcoidosis. Patients were recruited regardless of treatment, apparent extrapulmonary involvement and symptoms; written informed consent was obtained. To estimate extrapulmonary disease 